\title{
L'évolution de l'ordre des mots en français : Chronologie, périodisation, et réorganisation du système
}

\author{
Christiane Marchello-Nizia \\ ENS-Lettres et sciences humaines, Lyon, UMR «ICAR » \\ marchell@linguist.jussieu.fr
}

\section{Introduction}

Le statut du complément d'objet direct est différent, tant en synchronie qu'en diachronie, selon la perspective théorique que l'on adopte. Dans cette étude, nous montrerons que dans l'analyse de l'évolution de la syntaxe de la phrase en français, le comportement de l'objet direct nominal joue un rôle important.

Après avoir présenté un 'état de l'art' sur la question de l'évolution de la syntaxe phrastique en français, et rappelé les résultats désormais admis avec leur chronologie, nous centrerons notre analyse sur les spécificités de l'évolution du statut et du comportement de l'objet, dont la syntaxe est bien moins connue que celle du sujet et du verbe, tant en synchronie qu'en diachronie. Nous montrerons que les constructions dans lesquelles entre l'objet présentent des spécificités qui le distinguent des autres constituants, et dans cette perspective nous approfondirons la chronologie évolutive de deux constructions particulières. Nous nous demanderons ensuite si les changements qu'ont connus le sujet et le verbe sont liés entre eux d'une part, et à ceux qui ont touché l'objet d'autre part, ou bien si ces diverses modifications sont indépendantes et peuvent recevoir des explications différentes. Nous proposerons à partir des résultats obtenus de mettre au centre du dispositif explicatif la position de l'objet direct, et non comme habituellement le verbe ou le sujet.

Une telle démarche doit s'appuyer sur l'établissement d'une chronologie fine des changements, elle nécessite donc l'exploitation de données nombreuses et complexes, fondées sur l'analyse d'un corpus précis, mais aussi sur l'exploitation des résultats d'études antérieures.

\section{Le verbe et le sujet}

Une différence fondamentale bien connue entre le latin et le français moderne concerne la position des constituants de phrase que sont le sujet, le verbe et l'objet direct, et la mise en place de fortes contraintes pesant sur ces positions.

On rappellera brièvement l'état des connaissances concernant le sujet et le verbe, et on soulignera les questions pendantes.

\subsection{Le verbe}

Concernant l'évolution de la syntaxe du verbe, la question essentielle est celle de sa position dans la phrase : sa position la plus fréquente en ancien français est la seconde, après n'importe quel type de constituant, comme on le sait depuis les premiers travaux sur l'ancien français (Le Coultre 1875, Thurneysen 1892). Ce trait 'verbe second' (V2) a disparu par étapes, en moyen français, au profit d'une structure de type (XXX) SV (XXX).

Mais on doit affiner cette description, même si elle rend compte de la situation la plus fréquente. D'une part, contrairement aux langues strictement V2, l'ancien français a connu d'autres positions pour le verbe, aussi bien dans les phrases principales que dans les subordonnées. Dès les plus ancien textes, les phrases 
avec verbe en tête de phrase, ou au contraire en troisième ou quatrième position, ne sont pas exceptionnelles. Des études les ont décrites et en ont fourni une typologie (Moignet 1971).

Puis ad escole li bons pedre le mist. (Vie de saint Alexis, v. 33 : env. 1040)

En revanche, dans la prose du $13^{\mathrm{e}}$ s., les constructions en verbe initial se limitent quasiment aux cas où une principale suit une subordonnée :

Et por ce que je vi que li lyons estoit meins mesfesanz que li serpenz, corui je sus au serpent et l'ocis. (Queste del saint Graal p. 98).

Mais comme le souligne très justement Vance (1997 : 271), ce type de construction ne se rencontre plus au $15^{\mathrm{e}}$ s. En revanche, un autre type de structure en VX se développe et devient au $15^{\mathrm{e}} \mathrm{s}$. aussi fréquent qu'il l'était en très ancien français, tant en déclarative qu'en subordonnées (Vance 1997 : 261) :

Et ly direz que...(Saintré, p. 131)

Mais que soions en la chambre, nous rirons. (Saintré, p. 7)

Or selon Vance, il y a eu entre les deux étapes une réanalyse du sujet nul : en ancien français et encore au $13^{\mathrm{e}} \mathrm{s}$., cette construction était interprétée comme un sujet pronominal postposé effacé ; en moyen français et spécialement au $15^{\mathrm{e}} \mathrm{s}$, elle peut être interprétée de deux façons différentes, dans deux grammaires en compétition en cette période : soit comme la non expression d'ujn sujet postposé comme auparavant, soit, étant donné le développement du schéma dominant SV, comme la non-expression d'un sujet antéposé.

Donc entre le très ancien français et le français pré-classique, la position du verbe a connu plusieurs changements : d'abord V2 mais non strict puisque V1 ou V3 sont possibles, il devient lié au sujet qui en moyen français le précède majoritairement. Et selon Vance $(1997$ : 277)., le premier texte qui utilise la structure XSV - qui, comme l'avait montré Combettes (1988), est le signe du passage d'une structure V2 à une structure SV - de façon productive a été composé vers 1300 : c'est la Vie de saint Louis de Joinville.

Le français classique et le français moderne connaissent d'ailleurs encore la structure en V1 (Restait cette redoutable infanterie d'Espagne...), dont on examinera un sous-type dans la section 4.

\subsection{Le sujet}

Concernant la syntaxe du sujet, trois changements se sont produits : d'une part, la possibilité d'avoir un sujet nul a quasiment disparu - mais tardivement ; d'autre part, la nature du sujet pronominal a changé : de partiellement autonome, celui-ci est devenu nettement clitique vers 1400 ; enfin, la position du sujet nominal ou pronominal par rapport au verbe conjugué s'est progressivement fixée.

La régression du sujet nul est attestée par toutes les études qui comparent la syntaxe phrastique au cours des siècles.

En encore au milieu du $17^{\mathrm{e}}$ s., Vaugelas admet que, parfois, la suppression du pronom personnel est élégante (Fournier 1998 : p. 39) ; mais dès le début du $18^{\mathrm{e}}$ s., dans les Observations...sur les Remarques de M. de Vaugelas (1705) l'Académie française critique cette position : la suppression du pronom est devenu 'une faute'. Par aiklleurs, si la chronologie de la cliticisation du sujet pronominal a donné lieu à des analyses assez diverses (G. Zink 1997 la date de 1400 environ, Vance la situe bien plus tôt), il est hors de doute que ce changement a favorisé l'expression du sujet.

Parallèlement au développement de la contrainte d'expression du sujet, celle de sa position préverbale se développait également - sans que des arguments dirimants aient été apportés pour expliquer ce changement. Mais depuis le moyen français (Prévost 2001) et encore en français moderne (Fuchs 1997), il est des cas où la postposition du sujet au verbe s'impose, et d'autres où elle est possible.

Ces deux changements ont laissé percevoir qu'au cours des siècles la cohésion entre le verbe et son sujet s'est fortement développée en français. On se demandera ci-dessous si la cohésion du verbe et son sujet est supérieure ou non à celle du verbe et son objet direct. 


\section{$3 \quad$ L'objet direct nominal, ses spécificités}

\subsection{L'objet direct : des régions encore peu explorées}

En ce qui concerne l'objet direct, surtout nominal, la somme de nos connaissances est plus réduite. Plusieurs phénomènes expliquent cet état des choses.

Tout d'abord, parmi les très nombreuses études historiques sur l'ordre des mots en français, il en est peu qui aient accordé une importance particulière à la fonction d'objet direct. La plupart des analyses concernent le sujet et le verbe, et de façon générale surtout la périphérie gauche de la phrase (M. Adams 1987, F. Dupuis 1989, B. Vance 1997, Rouquier 2006). Ce n'est que sous l'influence de la typologie linguistique que cette situation s'est modifiée, et que quelques études récentes ont défriché le terrain (Marchello-Nizia 1995, Schoesler 2000, 2001). De façon générale d'ailleurs, la zone postverbale n'a guère été explorée. Et comme nous allons le voir, plusieurs aspects de l'évolution de sa syntaxe sont encore imparfaitement connus. Et par exemple, des phrases du type : Ensuite prirent place les ambassadeurs, restent inconnues de la plupart des grammaires, puisque l'objet n'est pas supposé séparer un verbe de son sujet.

Une seconde raison est que la syntaxe de l'objet pronom personnel ayant été bien décrite (notamment grâce à l'attention portée aux clitiques par la grammaire générative et par la syntaxe positionnelle (Skårup 1975), l'objet nominal a quant à lui été laissé dans l'ombre. L'une des plus récentes études sur l'ordre des mots en français médiéval, celle de Vance (1997), n'accorde aucune attention précise à la fonction syntaxique d'objet, qui n'est pas distingué d'autres constituants tels que l'attribut, le participe passé ou l'infinitif construits avec un auxiliaire ou un verbe modal, les compléments circonstanciels ou les adverbiaux inter- ou intraphrastiques.

Si l'on peut montrer que la syntaxe de l'objet direct nominal en français, et spécialement en ancien français, ne se différencie pas de celle des autres constituants non sujets, cette indifférence se justifierait. Mais en est-il ainsi ?

\subsection{L'objet nominal a-t-il la même syntaxe que les autres constituants non sujets ? ou bien a-t-il une syntaxe spécifique?}

Deux études faites dans deux optiques théoriques différentes vont nous permettre de répondre à cette question.

En 1995, nous avons effectué une analyse typologique de l'évolution de la syntaxe phrastique en français. Comme c'est de règle dans cette perspective qui se fonde sur la position relative des constituants fondamentaux que sont le verbe, le sujet et l'objet direct (Greenberg 1963, Vennemann 1974, Lehmann 1974), nous avions analysé la syntaxe des déclaratives, en focalisant notre commentaire sur l'objet nominal. Ayant choisi de comparer deux textes, nous avions choisi de travailler sur le dépouillement exhaustif de la Chanson de Roland (soit un peu plus de 1000 déclaratives à verbe trabnsitif et objet nominal) et de la Queste del saint Graal (soit 1727 déclaratives ayant ces mêmes spécificités). Dans ces deux textes, nous avions mis au jour les divers schémas de phrases déclaratives à objet direct nominal qui étaient représentés dans chacun de ces textes, de façon à identifier les schémas les plus fréquents à deux époques différentes.

Or, deux ans plus tard, en 1997, dans une perspective théorique très différente, celle des Principes et paramètres initiée en grammaire générative par D. Lightfoot, B. Vance a également analysé Queste del saint Graal. Mais dans son cadre théorique, l'objet, et spécialement l'objet nominal, ne bénéficie d'aucun statut théorique ; elle a donc sélectionné un corpus de 85 pages, comportant environ 3000 propositions tant principales que subordonnées (soit 1746 phrases indépendantes ou principales et 1345 subordonnées), et sans limiter son enquête aux verbes transitifs comme nous l'avions fait. 
Du même texte, la Queste del saint Graal, roman en prose écrit vers $1220^{1}$, nous avons ainsi deux descriptions certes très différentes, mais dont justement la différence va nous permettre de comparer la syntaxe de l'objet nominal d'une part, et des autres constituants que le sujet d'autre part (au nombre desquels, entre autres, l'objet nominal).

Ces deux séries de résultats se différencient donc doublement: d'une part par le fait que les nôtres ne comptaient que l'objet nominal comme élément autre que le verbe et le sujet, alors que ceux de Vance prennent en compte tous les autres constituants quels qu'ils soient, qui sont symbolisés par X; et d'autre part par le fait que nous n'avions sélectionné que les déclaratives, alors que Vance a pris en compte également les subordonnées. Afin de rendre la comparaison possible, nous ne conserverons des résultats obtenus par Vance que ceux concernant les principales (main sentences), puisque notre analyse typologique les prenait seules en compte.

En comparant ces deux séries de chiffres, nous examinerons si les chiffres concernant l'objet nominal (selon Marchello-Nizia 1995) et ceux concernant plus généralement les constituants autres que le sujet (attributs, objets, circonstants, adverbes, connecteurs...) (selon Vance 1997) coïncident ou non. Si ces chiffres sont proches ou très proches, c'est que le comportement de l'objet nominal est semblable à celui des autres constituants - auquel cas nous pourrons conclure que l'objet nominal ne possède aucune particularité syntaxique propre. Si en revanche les chiffres révèlent une distorsion, c'est que la syntaxe de l'objet direct nominal possède une spécificité.

Les éléments de cette comparaison sont représentés dans le tableau ci-dessous.

\begin{tabular}{|c|c|c|c|c|}
\hline Schémas de phrases & \multicolumn{2}{|c|}{$\begin{array}{c}\text { Marchello-Nizia (1995) } \\
\text { (1727 phrases) }\end{array}$} & \multicolumn{2}{|l|}{$\begin{array}{l}\text { Vance (1997) } \\
(1746 \text { phrases })^{2}\end{array}$} \\
\hline $\begin{array}{l}\text { Schéma en SVX et } \\
\text { SVOn: } \\
\text { sujet antéposé au verbe }\end{array}$ & SVOn : 622 & $36 \%$ & SVX : 799 & $46 \%^{3}$ \\
\hline $\begin{array}{l}\text { Schéma en CVS et OVS : } \\
\text { sujet postposé au verbe }\end{array}$ & OnV S : 38 & $2 \%$ & CVS : 306 & $17,5 \%$ \\
\hline $\begin{array}{l}\text { Schéma en } \mathrm{CV} \text { et } \mathrm{OV} \text { : } \\
\text { sans sujet exprimé }\end{array}$ & OnV (avec sujet zéro) : 18 & $1 \%$ & $\begin{array}{l}\text { CV (avec sujet nul) : } \\
576\end{array}$ & $33 \%$ \\
\hline Autres schémas: & $\begin{array}{l}\text { CVOn }: 865 \\
\text { CVOnSn }: 3 \\
\text { CVSOn }: 181\end{array}$ & $\begin{array}{l}50 \% \\
- \\
11 \%\end{array}$ & & \\
\hline
\end{tabular}

Si la syntaxe de l'objet nominal était comparable à celle de n'importe quel autre constituant non sujet, on s'attendrait à trouver pour chacune des constructions exqaminées des chiffres comparables. Or ce n'est pas le cas. Ce tableau montre une nette différence entre l'attitude de l'objet nominal et celle des constituants regroupés par Vance sous les symboles $\mathrm{C}$ ou X.

Certes, dans les deux séries de colonnes il apparaît que, quelle que soit la nature du verbe (transitif ou non), le schéma le plus fréquent dans les déclaratives consiste à placer avant le verbe non pas le sujet, mais un autre constituant.

Si la première ligne de résultats ne fait pas apparaître une distorsion criante, les lignes suivantes en revanche révèlent où se situe la différence essentielle : alors que les arguments autres que le sujet, tous confondus, se placent majoritairement avant le verbe, l'objet, si on l'extrait de ce groupe, révèle une attitude très dissidente : alors que plus de $50 \%$ des déclaratives commencent par l'un de ces constituants, 
ce n'est que dans 3\% des déclaratives que ce constituant initial autre que le sujet se trouve être l'objet nominal.

Une deuxième différence, très significative à notre avis, est la suivante : l'absence de sujet est assez nettement supérieure dans les déclaratives à verbe transitif et objet nominal $(51 \%$ si à CVOn on ajoute OnV) que dans l'ensemble des déclaratives, tous types de verbes confondus. Cela signifie que la structure habituelle d'une déclarative en ancien français, dans la prose du $13^{\mathrm{e}} \mathrm{s}$., est la présence d'un argument nominal du verbe et d'un seul - qu'il s'agisse du sujet ou du verbe.

On peut dès lors mieux interpréter la troisième différence que révèle le tableau, et qui, elle, joue sur de petits nombres : lorsque l'objet direct nominal est antéposé au verbe et en tête de phrase (ce qui ne concerne que $3 \%$ des déclaratives), en général le sujet est également exprimé. Cela peut paraître surprenant après ce que l'on vient de dire concernant le fait qu'un seul argument essentiel suffit auprès du verbe - qui sera interprété comme sujet si le verbe est intransitif, et objet s'il est transitif (Schoesler 1984 ????). En fait, que ces phrases à objet initial comportent également le second argument ne fait que renforcer leur caractère marqué, que montre d'une part leur rareté (3\% ees déclaratives) et d'autre part l'analyse qu'on a pu en donner (Marchello-Nizia 1995 : 90-99).

Quoi qu'il en soit, même si l'analyse que l'on vient de mener ne concerne que les déclaratives, il est clair que dans ce type de proposition au moins (qui sont les plus fréquentes), l'objet nominal possède clairement une spécificité syntaxique.

\section{L'évolution de la syntaxe de l'objet en français}

\subsection{Quelques rappels}

1) L'évolution syntaxique de l'objet nominal en français est caractérisée par cinq traits : une contrainte presque absolue, une tendance forte et trois changements :

2) l'objet pronom personnel est cliticisé, antéposé et contigu au verbe très majoritairement dès le plus ancien français ;

3) l'objet nominal était antéposé au verbe dans les déclaratives dans les plus anciens textes (Herman 1954/1990: 239); c'est le cas dans Jonas. Mais dès le $11^{\mathrm{e}} \mathrm{s}$. il se postpose majoritairement, et au $12^{\mathrm{e}} \mathrm{s}$. il est toujours majoritairement postposé au verbe,

4) et cette tendance est devenue une contrainte forte dès le 13e s. (Marchello-Nizia 1995) - sans doute comme résultat du passage d'un 'branchement à gauche' vers un 'branchement à droite' (tendance qui existait semble-t-il dès le latin classique : J. N. Adams 1976, Bauer 1995);

5) l'inversion d'une optionalité : en ancien français, le sujet était optionnel mais pas l'objet, en français moderne c'est l'objet qui est devenu optionnel (Lambrecht \& Lemoine 1996, Larjavaara 2001, Schoesler 2001) alors que le sujet est obligatoirement exprimé ;

et - c'est ce que nous allons montrer plus précisément ci-dessous en 4.2 et 4.3 -l'établissement progressif d'une obligation de contiguïté entre l'objet nominal et le verbe.

\subsection{La cohésion entre l'objet nominal et le verbe est-elle plus faible ou plus forte que celle qui lie le sujet et le verbe $?^{4}$}

La contrainte de contiguité entre deux constituants peut être interprétée comme la marque d'une cohésion forte entre ces éléments. Pour vérifier le degré de cohésion entre l'objet et le verbe, nous le comparerons à celui qui lie le sujet et le verbe.

Il existe des constructions qui permettent de mener facilement cette comparaison : ce sont celles où l'objet nominal et le sujet se trouvent du même côté du verbe. La combinaison de $\mathrm{S}$ et $\mathrm{O}$ ensemble avant 
ou après le verbe donne quatre possibilités : SOV, OSV, VSO, VOS. Toutes ces constructions existaient en ancien français, mais seule la dernière encore en français moderne. Il s'agit des schémas suivants : SOnV, OnSV, (X)VSOn, (X)VOnS. On examinera le développement de ces structures et les étapes de leur changement.

Pour l'ancien français, Foulet avait bien repéré, entre autres possibilités, ces quatre schémas (1930/1967 : § 36-44, et $\S 49-58)$. Il qualifie OSV et VOS de rares, et donc n'analyse que les deux autres, SOV et VSO - qui, soulignons-le, présentent l'ordre SO, infiniment plus fréquent dans les langues du monde que l'ordre OS des deux autres schémas (Greenberg 1963, Lehmann 1974).

En ancien français, la structure $\operatorname{SOV}$ (réalisée en $\operatorname{SpOnv}(\mathrm{X})$ ou $\operatorname{SnOnV}(\mathrm{X})$ ) est attestée dès le $9^{\mathrm{e}}$ s. avec un $\mathrm{Sp}$, dès le $10^{\mathrm{e}} \mathrm{s}$. avec un $\mathrm{Sn}$ :

Elle colpes non auret (Eulalie 20 : 'Elle n'avait commis aucun péché')

Li serf sum pedre...La lavadures li getent sur la teste (Vie de saint Alexis 263-4 : 'Les serviteurs de son père les eaux sales lui jettent sur la tête' = Les serviteurs de son père lui jettent sur la tête les eaux sales')

Le pronom personnel sujet est possible dans cette construction au moins jusqu'au milieu du $12^{\mathrm{e}} \mathrm{s}$. dans les déclaratives, un peu plus longtemps dans les subordonnées. Quant à la structure SnOnV..., elle apparaît encore en moyen français, et n'est pas rare chez certains auteurs. Elle est encore employée au début du $17^{\text {e }}$ s. :

Et si quelque bonheur nos armes accompagne,.. (La Fontaine, 1678)

Le second schéma, OSV (X), est qualifié de rare par Foulet (1930/1967 : 39), Schoesler (1984 : 224), et Buridant (2000). Il se trouve cependant employé tout au long des $12^{\mathrm{e}}-15^{\mathrm{e}} \mathrm{s}$., tant avec un sujet pronominal qu'avec un sujet nominal. Dans les cas relevés, il s'agit soit d'emphatiser l'objet nominal, qu'il soit rhématique ou thématique. Ainsi au $12^{\mathrm{e}} \mathrm{s}$ : :

Nule riens je n'i donroie. (Colin Muset, Chansons VII 14)

Sire, fet il, amistié grande / Mesire Guillaume vos mande. (Huon le Roi, Le vair palefroi 1289-90)

Le nun de traïtur seinz Thomas n'entendi. (Guernes, Vie de saint Thomas Becket, 5506)

Cette construction perdure longtemps, spécialement avec un sujet pronom personnel, que ce soit en déclarative ou en subordonnée (comme par exemple ; chez Christine de Pizan) :

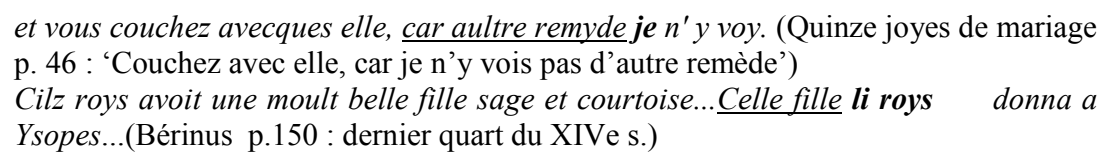

Le troisième schéma, où sujet et objet sont postposés au verbe dans l'ordre VSO, est attestée dès le $11^{\mathrm{e}} \mathrm{s}$., et perdure encore jusque dans la première moitié du $17^{\mathrm{e}} \mathrm{s}$. (La Fontaine), mais Vaugelas la refuse totalement. Dans la période médiévale, c'est la plus fréquente des quatre construction examinées ici :

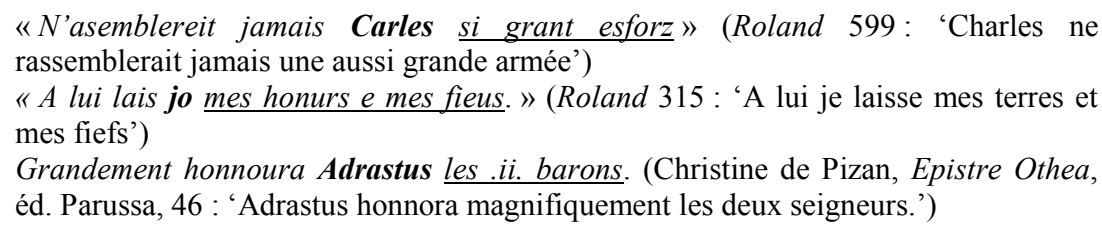

Quant au dernier schéma, (X)VOS, il n'est possible qu'avec un sujet nominal, comme l'a montré Skårup dans le cadre de la théorie positionnelle (1975). Il se rencontre dès le $11^{\mathrm{e}} \mathrm{s}$. :

En sum puing tint le cartre le Deu serf. (Vie de saint Alexis 348 : Dans sa main le serviteur de Dieu tenait la lettre') 
$\mathrm{Au} 17^{\mathrm{e}} \mathrm{s}$, , ni les grammairiens ni les remarqueurs n'évoquent cette structure (à ma connaissance), mais elle n'est pas rare, avec les mêmes contraintes qu'en français moderne : avec un 'verbe support', ou avec sujet lourd (nous avons effectué des recherches dans FRANTEXT autour du groupe 'prendre (...) place') :

Ici prend sa place le glorieux Martyr Monsieur Sainct Biron, qui ayant vescu en mespris de toute religion..(Agrippa d'Aubigné, Confession catholique.., 1630, livre 2, p. 364)

La seule étude consacrée à ce schéma de phrase est celle de Valois \& Dupuis (1992) sur l'inversion stylistique en subordonnée ${ }^{5}$, où les auteurs sont conduits à examiner entre autres ce cas. Et pourtant les exemples ne sont pas exceptionnels ${ }^{6}$, en déclarative ou en subordonnée, avec ou sans un élément introducteur :

\author{
Paieront une amende tous les automobilistes en infraction. \\ Ne tolèrent pas non plus cette place en général les adverbes correspondant à une \\ recatégorisation (D. Leeman, à par.) \\ Se firent jour alors deux priorités,... \\ Ensuite prirent (leur) place les autres invités / les retardataires.
}

La construction qui a été conservée est une construction paradoxale, où le sujet comme l'objet ne sont pas dans la position habituelle : le sujet est postposé au verbe et en est séparé ; et l'objet quant à lui, s'il est postposé au verbe comme attendu, sépare le verbe de son sujet, ce qui est n'est pas habituel.

En français moderne, les emplois de cette construction semblent avoir deux caractéristiques : ce sont des énoncés totalement rhématiques, qui se rencontrent soit dans des textes à caractère administratif et déontique, soit dans des narrations où plusieurs actions s'enchaînent (l'effet 'liste').

Au plan syntaxique, Valois \& Dupuis concluaient, au terme de leur étude menée dans le cadre de la théorie générative : "Clearly some type of adjacency must be observed between the verb in Infl and the object in VP." (1992: 332). Nous reprendrons cette idée de cohésion et expliquons cette adjacence forte entre $\mathrm{V}$ et $\mathrm{O}$, plus forte que celle entre $\mathrm{V}$ et $\mathrm{S}$, en proposant de voir dans cette séquence le fait que la solidarité syntaxique entre $\mathrm{V}$ et $\mathrm{O}$ est plus forte que celle entre $\mathrm{S}$ et $\mathrm{V}$, au moins depuis le français préclassique, époque à laquelle ont disparu les constructions où l'objet nominal était séparé du verbe par le sujet nominal.

\title{
4.3 L'évolution de la position de l'objet direct nominal en français
}

En très ancien français, la position de l'objet direct nominal est variable : il s'antépose fréquemment au verbe. Ainsi dans le plus ancien texte en français, les Serments de Strasbourg, il n'y a guère que deux déclaratives à comporter un objet nominal : l'une offre l'objet antéposé, l'autre postposé :

\author{
Et ab Ludher nul plaid nunquam prindrai...(serment prononcé par Louis, éd. Wagner- \\ Collet) \\ Si salvari eo cist meon fradre Karlo (serment prononcé par Louis, éd. Wagner-Collet)
}

Dans la Séquence de sainte Eulalie, sur 7 déclaratives à verbe transitif et objet nominal, 2 antéposent l'objet au verbe, 5 le postposent.

Au milieu du $10^{\mathrm{e}}$ s.(vers 940-950), dans le Sermon sur Jonas (ou Fragment de Valenciennes), brouillon d'un texte de sermon qui mêle français et latin, dans les parties nettement en français l'objet est antéposé au verbe dans 9 propositions sur 10 (Herman 1954/1990 : 239).

En revanche, dès le $11^{\mathrm{e}} \mathrm{s}$, dans la Vie de saint Alexis, l'objet nominal est déjà postposé au verbe dans 67\% des cas (Völcker 1882:30). Et de même dès le début du $12^{\mathrm{e}}$, dans la Chanson de Roland, dans les déclaratives à verbe transitif, l'objet direct nominal est postposé dans 66\% des cas (Marchello-Nizia 1995 : 72-79). Quelques décennies plus tard, dans le ms. C des Psaumes, dans 22 cas sur 25, un complément d'objet direct antéposé en latin est postposé dans la traduction française (Herman p. 256). 
Un siècle plus tard, en prose, la situation a largement changé. Dans la Conquête de Constantinople de Villehardouin, Siepmann (1937: 15) a relevé seulement 22 cas d'antéposition de l'objet sur 704 propositions (tous types confondus) ayant un verbe transitif et un objet nominal, soit $4,3 \%$ des cas - alors que s'agissant du sujet, les cas de postposition s'élevaient à 40\%. Dans la Queste del saint Graal (datée d'env. 1220), dans les déclaratives à verbe transitif et objet nominal, ce dernier est postposé au verbe dans 97\% des cas, les 3\% des cas d'antéposition étant nettement caractérisables (Marchello-Nizia 1995 : 79 84). Et au tournant des $13^{\mathrm{e}}$ et $14^{\mathrm{e}}$ s., dans la Vie de saint Louis de Joinville, G. Marx (1881 : 347) relève en principale $89 \%$ d'objets nominaux postposés au verbe.

En moyen français, dans un roman en prose composé vers 1375, Berinus, B. Lewinski (1949: 84-85) évalue à $97,1 \%$ la proportion d'objets nominaux postposés au verbe en déclaratives, et 92,1 en subordonnée. Dans la Chronique de Jean d'Outremeuse, les chiffres correspondant relevés par H. Nissen (1943 : 83) sont de $95,1 \%$ et $94,1 \%$ respectivement. Et tous les textes analysés par la suite offrent des proportions comparables.

On peut donc en conclure que dès le début du $13^{\mathrm{e}}$ s., en prose, la position 'moderne' de l'objet direct nominal est déjà fixée, et qu'elle ne variera plus - ce qui, on l'a vu, n'est pas le cas du sujet et du verbe, dont les variations positionnelles durent plus longtemps.

\subsection{Jusqu'à quand l'objet nominal peut-il précéder le verbe en subordonnée relative?}

Il est connu qu'en ancien français, les subordonnées ne suivent pas exactement la même syntaxe que les déclaratives. Certains types de subordonnées, par exemple, présentent l'ordre SVOn dans un fort pourcentage de cas.

Mais d'autres offrent au contraire une syntaxe plus archaïque que les déclaratives, puisqu'elles adoptent souvent l'ordre OnV :

Tholomer, qui sa terre li demandoit (Queste del saint graal, p. 32)

Et comme l'avait bien montré Dupuis et l'a confirmé Vance (1997), les relatives en QUI sont parmi les plus 'archaïsantes' des subordonnées. Ainsi, sur 500 relatives en QUI relevées dans les cent premières pages de l'édition Pauphilet de la Queste del saint Graal (le texte qui nous a servi pour la comparaison effectuée en 3.3 ci-dessus), 107 ont un verbe final, soit plus de $20 \%$ d'entre elles.

Nous avons relevé dans les mêmes cent premières pages de la Queste del saint Graal, une centaine de relatives en QUI ayant un verbe transitif avec un objet direct nominal. Sur ces 100 relatives ayant donc un sujet initial, 73 présentent l'ordre V-On, mais 27 ont On-V, avec le plus souvent le verbe final. 27\% de ces relatives ont donc un ordre archaïque, à la différence des déclaratives dont seulement 3\% plaçaient l'objet nominal avant le verbe :

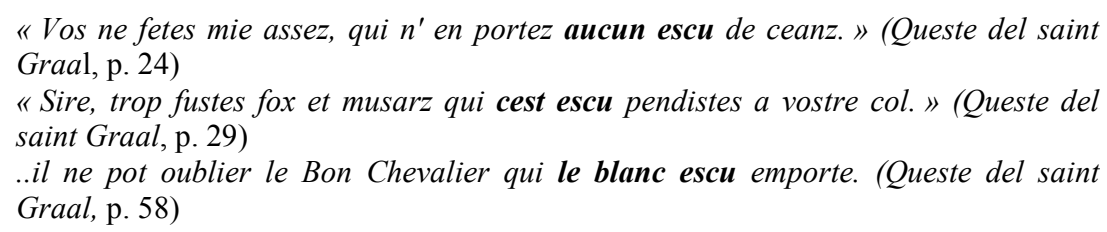

Moins d'un siècle plus tard, cet ilot de résistance a presque disparu : ces relatives 'archaïsantes' ont rejoint la syntaxe désormais commune. Chez Joinville, vers 1300, sur 500 relatives en QUI relevées entre les p. 2 et 182 de l'édition et traduction réalisées par J. Monfrin, seulement 43 présentent un verbe final, soit 8,5\%. Dans bien des cas, il s'agit de relatives formulaires telles que qui la estoient, qui lors vivoient, qui mort estoient.

Et dans ce même corpus, sur une centaine de relatives en QUI ayant un verbe transitif et un objet nominal, il ne s'en trouve plus que 9 , c'est à dire $9 \%$, à placer l'objet nominal avant le verbe - que celui-ci soit final ou non : 


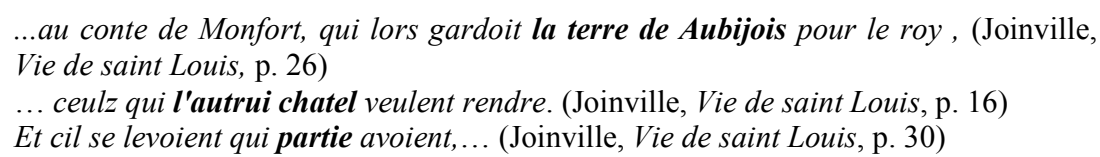

Et chez Commynes, dans son dernier livre, la proportion des relatives en QUI présentant un verbe final précédé d'un complément a encore baissé : seules $5 \%$ ont cette construction :

$$
\text { ...le bailly de Victry, qui bien se y conduisit. (Commynes, Mémoires, 8, p. 5) }
$$

Mais à cette période de la fin $\mathrm{du} 15^{\mathrm{e}}$ et du début du $16^{\mathrm{e}}$ s., l'objet nominal a totalement cessé de s'antéposer : aucune des 550 relatives en QUI de ce Livre ne présente plus cette construction, et ce n'est que dans quelques très rares cas que le quantifieur tant précède le verbe, mais le reste du syntagme objet suit :

en ceste maison, qui tant avoit receü de gloire et d' honneur, (Commynes, Mémoires, 8, p. 161)

\section{Chronologie des changements syntaxiques, éléments d'explication}

Dans cette étude, nous avons mis en évidence la spécificité syntaxique de la fonction objet nominal sous forme nominale. Nous avons également montré que la position postverbale de l'objet nominal est quasiment acquise dans les déclaratives au début du $13^{\mathrm{e}}$ s. en prose, et dans cet îlot de résistance que sont les relatives en qui dès la fin du $15^{\mathrm{e}} \mathrm{s}$. Il faudra confirmer cette analyse par l'examen de textes en vers, et vérifier que cette syntaxe vaut dans des genres textuels variés. Le parsage syntaxique en cours de textes nombreux permettra cette enquête. En troisième lieu, on a vu également que la cohésion entre le verbe et l'objet nominal est plus forte que celle qui lie le verbe à son sujet.

Ces divers éléments concourent à mettre en lumière l'importance et la précocité de l'évolution syntaxique de l'objet nominal, au point que, dans une étape ultérieure, nous tenterons de proposer une alternative à l'explication de cette évolution de l'ordre des mots simplement par la perte de la position seconde du verbe et l'expression d'un sujet antéposé au verbe. Le développement d'une structure dominante SVO pourrait être interprétée comme le résultat d'une première étape où, après que l'objet pronominal s'est fixé devant le verbe, l'objet nominal à son tour s'est fixé après le verbe, la position initiale étant réservée au constituant thématique.

\section{Références bibliographiques}

Adams, M. P. (1987). Old French, Null Subjects and Verb Second Phenomena. Ph. D. : U. C. L.A.)

Buridant, C. (2000). Grammaire nouvelle de l'ancien français. SEDES : Paris.

Combettes, B. (1988). Recherches sur l'ordre des éléments de la phrase en moyen français, Thèse pour le Doctorat d'Etat, Université de Nancy; exemplaire dactylographié.

Dupuis, F. (1989). L'expression du sujet dans les subordonnées en ancien français. PhD Université de Montréal.

Foulet, L. (1930/1967). Petite syntaxe de l'ancien français. Paris : Champion.

Fournier, N. (1998). Grammaire du français classique, Paris : Belin-Sup.

Fournier, N. (2001). Expression et place des constituants dans l'énoncé en français classique : la relation sujet-verbe et la relation verbe-objet. Langue française 130, pp. 89-107.

Fuchs, C. ed. (1997). La place du sujet en français contemporain. Louvain : Duculot.

Haase, A. $\left(1969^{7}\right)$. Syntaxe française du XVIIe s. Paris : Delagrave. 
Herman, J. (1954/1990). Recherches sur l'ordre des mots dans les plus anciens textes français en prose. Acta linguistica Academiae Hungaricae IV, p.69-93 et 351-379 (repris in Herman, J. 1990. Du latin aux langues romanes, Etudes de linguistique historique réunies par S. Kiss. Tübingen: Max Niemeyer Verlag).

Lambrecht, K. \& Lemoine, K. (1996). Vers une grammaire des compléments d'objet zéro en français parlé. Travaux linguistiques du CerLiCO/9, 270-310.

Larjavaara, M. (2000). Présence ou absence de l'objet: limites du possible en français contemporain. Academia Scientiarum Fennica.

Lewinski, B. (1949). L'ordre des mots dans Bérinus, roman en prose du XIVe siècle. Göteborg.

Marchello-Nizia, C. (1995). L'évolution du français : ordre des mots, démonstratifs, accent tonique. Paris : Armand Colin.

Marx, G. (1881). Über die Wortstellung bei Joinville. Heilbronn.

Moignet, G. (1971). L’ordre verbe-sujet dans la Chanson de Roland. Mélanges Boutière, t. I, p. 397-421.

Nissen, H. (1943). L'ordre des mots dans la Chronique de Jean d'Outremeuse. Uppsala.

Prévost, S. (2001). La postposition du sujet en français aux XVe et XVIe siècles. Paris, Editions du CNRS

Price, Gl. (1961). Aspects de l'ordre des mots dans les Chroniques de Froissart. Zeitschrift für romanische Philologie 77, 15-48.

Rodriguez Somolinos, A. (1983). Estudio distribucional del sujeto en Villehardouin y en Froissart, Tesis doctoral, Madrid.

Rouquier, M. (2006). L'expression et la position des sujets pronominaux et lexicaux dans La Vie de saint Léger et La Passion du Christ. L'Information grammaticale 110, 3-8.

Schoesler, L. (2000). Permanence et variation de la valence verbale : réflexions sur la construction des verbes en latin, en ancien français, en moyen français et en français moderne. Les nouvelles ambitions de la linguistique diachronique, Actes du XXIIe Congrès International de Linguistique et de Philologie Romanes 1998. Englebert, A., Pierrard, M., Rosier, L. and Van Raemdonck D. (eds), 407418. Max Niemeyer.

Schoesler, L. (2001). The coding of the subject-object distinction from Latin to Modern French. Grammatical Relations in Change, ed. By Faarlund, J. T. Amsterdam-Philadelphia, John Benjamins, 273-302.

Siepmann, E. (1937). Die Wortstellung in der Conquête de Constantinople de Villehardouin. Bochum.

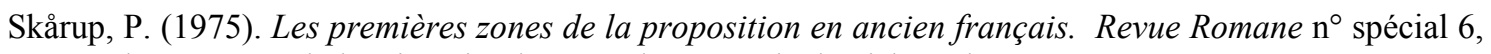
Etudes romanes de l'Université de Copenhague : Akademisk Forlag.

Valois, D. \& Dupuis, F. (1992). On the status of (verbal) traces in French: The Case of stylistic inversion. Romance Languages and Modern Linguistic Theory. Amsterdam \& Philadelphia: John Benjamins. 325-338.

Vance, B. (1997). Syntactic Change in Medieval French: Verb-Second and Null Subjects. Dordrecht: Kluwer Academic Publishers.

\footnotetext{
${ }^{1}$ Et dans la même édition, celle de Pauphilet (Champion).

${ }^{2}$ Ces chiffres sont tirés du Tableau 3.2 (p. 38) de Vance (1997).

3 "SVX order occurs in about 46\% of the main clauses counted in our sample of the Queste.." (Vance 1997: 38).

${ }^{4}$ Une partie de l'analyse présentée dans cette section est issue d'un article à paraître.
} 
ISBN 978-2-7598-0358-3, Paris, 2008, Institut de Linguistique Française

Conférences plénières

DOI $10.1051 / \mathrm{cmlf08342}$

${ }^{5}$ On the status of (verbal) traces in French : The Case of stylistic inversion.

${ }^{6}$ En français d'autres éléments de la valence verbale (compléments indirects ou de direction, attribut du sujet, Vpp) ont la même possibilité de se construire ainsi : Sont à ce titre archä̈ques les exemples suivants (N. Fournier 1998: 36) ; A ceux qui ont les plus hauts salaires seront demandés les plus gros efforts. (cp : A celui qui a le plus reçu sera le plus grand compte demandé Pascal, cité par Haase § 153). 